\title{
COMPLEX MAGNETIC STRUCTURE OF STRONGLY COUPLED Fe/Si MULTILAYERS
}

\author{
J. Dubowik ${ }^{a}$, F. StobieCKi ${ }^{a}$, B. SzYmański ${ }^{a}$, YU.V. KudRYAVtSEV $^{b}$, \\ A. GrabIas ${ }^{c}$ and M. KoPCEWICZ ${ }^{c}$ \\ ${ }^{a}$ Institute of Molecular Physics, Polish Academy of Sciences \\ Smoluchowskiego 17, 60-179 Poznan', Poland \\ ${ }^{b}$ Institute of Metal Physics, National Academy of Sciences of Ukraine \\ Kiev-142, Ukraine \\ cInstitute of Electronic Materials Technology, Wólczyńska 133, 01-919 Warsaw, Poland
}

$\mathrm{Fe} / \mathrm{Si}$ multilayers with strong bilinear and biquadratic couplings were investigated. A complex structure revealed by the Mössbauer spectroscopy corresponds to multimode ferromagnetic resonance spectra in a non-saturated state. Simple dispersion relations for antiferromagnetic coupled bilayer structures are shown to be inapplicable to the $\mathrm{Fe} / \mathrm{Si}$ multilayers with a strong biquadratic component to the antiferromagnetic bilinear coupling.

PACS numbers: 75.70.-i, 68.55.-a, 76.50.+g

$\mathrm{Fe} / \mathrm{Si}$ layered structure represents a unique exchange coupled system in which a non-magnetic spacer is spontaneously created by a solid-state reaction during deposition process. Using various experimental techniques the Eindhoven group [1-4] concluded that $\mathrm{Si}$ spacer reacts with $\mathrm{Fe}$ creating a metallic, crystalline $\mathrm{Si}_{\mathbf{5} 0} \mathrm{Fe}_{50}$ which, in a specific way, mediate the coupling. Except for a strong, bilinear antiferromagnetic (AF) coupling, there is a substantial, apparent biquadratic component which is ascribed to a depth-dependent inhomogeneous structure of Fe/Si. To obtain information on a possible inhomogeneous magnetic structure inherent to the spontaneous solid-state reaction between $\mathrm{Fe}$ and $\mathrm{Si}$, we have extensively applied conversion-electron Mössbauer spectroscopy (CEMS) ạ̣d ferromagnetic resonance (FMR).

The $\mathrm{Fe} / \mathrm{Si}$ multilayers were prepared by rf-sputtering onto glass substrates. The individual Fe layer thicknesses were fixed at $t_{\mathrm{Fe}}=30 \AA$ whereas the nominal thicknesses of Si layer varied between $8 \AA$ and $22 \AA$ in $\left[t_{\mathrm{Fe}} \mathrm{Fe} / t_{\mathrm{Si}} \mathrm{Si}\right]_{N}$ layered structures with the number of repetitions $N=50$. The structural characterization was performed by low-angle and high-angle X-ray diffraction (XRD) using $\mathrm{Cu} K_{\alpha}$ radiation and the data obtained is in full agreement with earlier $\mathrm{X}$-ray studies [5].

The magnetic properties were studied using a vibrating sample magnetometer (VSM), C亡MS and FMR at 9.4 and $33.6 \mathrm{GHz}$ at room temperature. In-plane hysteresis loops for a series of $\left[30 \AA \mathrm{Fe} / t_{\mathrm{Si}} \mathrm{Si}\right]_{50}$ show a high saturation field peaked 
at $H_{\mathrm{S}}=10-12 \mathrm{kOe}$ at $t_{\mathrm{Si}}=13 \AA$ evidencing a strong AF exchange coupling. The convex shape of the magnetization loops suggests a substantial biquadratic contribution $J_{2}$ to the interlayer exchange energy $J_{1} M_{1} \cdot M_{2}+2 J_{2}\left(M_{1} \cdot M_{2}\right)^{2}$, where $J_{1}$ is the bilinear exchange constant, $\boldsymbol{M}_{1}$ and $\boldsymbol{M}_{2}$ are the magnetizations of the adjacent magnetic layers. Fitting the theoretical magnetization loops, calculated by minimizing the total magnetic energy of the multilayers, to the experimental data gives $J_{1}$ and $J_{2}$. For example, $J_{1}=0.44 \mathrm{erg} / \mathrm{cm}^{2}, J_{2}=0.09 \mathrm{erg} / \mathrm{cm}^{2}$ for a $[30 \AA \mathrm{Fe} / 15 \AA \mathrm{Si}]_{50}$ multilayer. For thinner nominal Si spacer, the biquadratic contribution is comparable to the bilinear term: for $t_{\mathrm{Si}}=11 \AA, J_{1}=0.62 \mathrm{erg} / \mathrm{cm}^{2}$ and $J_{2}=0.52 \mathrm{erg} / \mathrm{cm}^{2}$.

CEMS which is sensitive to the local order of iron atoms provided information on the composition of $\mathrm{Fe} / \mathrm{Si}$ multilayer structure. The data consists of complex spectra which can be related to (i) two non-magnetic components (about $25 \%$ of total $\mathrm{Fe}$ ), (ii) a magnetically split, six-line subspectrum about three times broader than that of $\alpha$-Fe suggesting a wide range of existing iron-rich silicides (50\%) and (iii) a component with hyperfine field characteristic of $\alpha$-Fe (25\%). The first non-magnetic component ( $8 \%$ of total $\mathrm{Fe}$ ) with the quadrupole splitting of about $0.4 \mathrm{~mm} / \mathrm{s}$ and the isomer shift (IS) of about $0.2 \mathrm{~mm} / \mathrm{s}$ can be related to a crystalline, metastable $\mathrm{Si}_{50} \mathrm{Fe}_{50}$ phase with $\mathrm{CsCl}$ structure [6]. The second single line (15\%) non-magnetic component with IS $=-0.04 \mathrm{~mm} / \mathrm{s}$ is of unknown origin. The relative intensity of the constituent spectra changes with thickness of $\mathrm{Si}$; for $8 \AA \mathrm{Si}$ no $\mathrm{Si}_{50} \mathrm{Fe}_{50}$ can be seen and for $22 \AA \mathrm{Si}$, about $21 \%$ of total $\mathrm{Fe}$ is in $\mathrm{Si}_{50} \mathrm{Fe}_{50}$ phase. In conclusion, the strongly $\mathrm{AF}$ coupled $\mathrm{Fe} / \mathrm{Si}$ system represents a layered structure with partially alloyed ferromagnetic Fe layers and inhomogeneous crystalline non-magnetic iron silicide spacers.

In-plane FMR measurements were done both in the non-saturated (X-band $-9.4 \mathrm{GHz})$ and nearly saturated $(Q$-band $-33.6 \mathrm{GHz})$ states to study the changes of magnetic structure of $\mathrm{Fe} / \mathrm{Si}$ on $t_{\mathrm{Si}}$ as well as the evolution of magnetization configuration with the external magnetic field. Contrary to the results obtained by Inomata et al. [7] who reported FMR investigations of relatively weakly coupled $\mathrm{Fe} / \mathrm{Si}$ multilayers, X-band FMR spectra of our samples are quite complicated revealing a large spread of the magnetic parameters across the film thickness. Generally, in the region of maximal AF coupling strength our X-band spectra consist of three modes: the fundamental mode and two higher order modes, one of which lies on the high and another one on the low field side of the fundamental mode as is shown in Fig. 1. The insert in Fig. 1 shows a typical FMR spectrum of the $[30 \AA \mathrm{Fe} / 13 \AA \mathrm{Si}]_{50}$ multilayer. The high field mode at about $2500 \mathrm{Oe}$, characteristic of AF coupling, is of very low intensity as compared to the low field (900 Oe) mode characteristic of the F coupled regions, with the intensity (depending on $t_{\mathrm{Si}}$ ) comparable to that of the fundamental mode. Such a behavior, i.e. the low field mode of a comparable intensity to the fundamental mode and an optic mode of negligible intensity, implies the presence of a mixed F/AF coupling.

Usually the AF coupling strength is determined from the position of a weak optic mode [7]. We, however, have found it impossible to relate the position of the high field mode to the substantial AF coupling strength in a similar manner. We found that the position of the fundamental mode, which may be regarded as an 


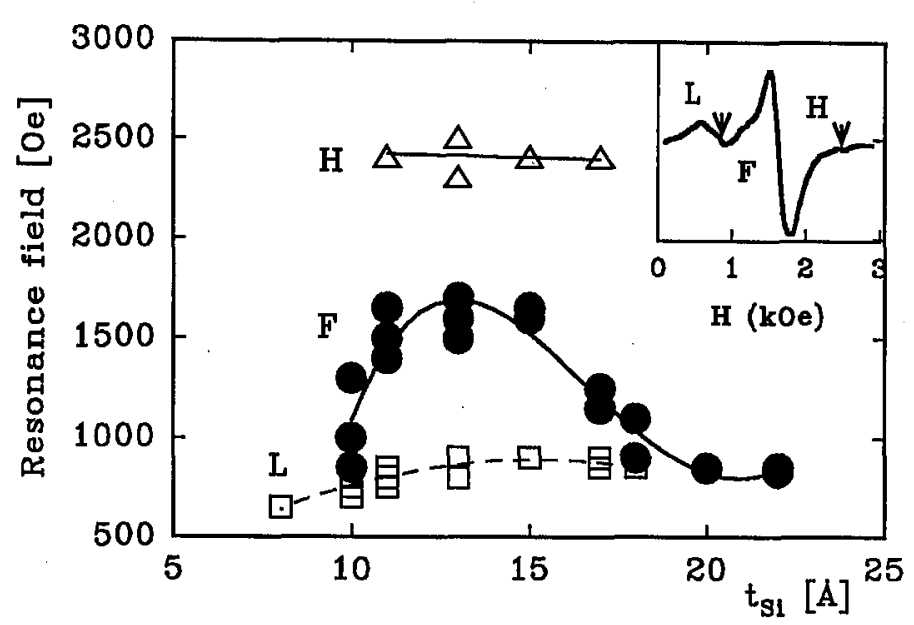

Fig. 1. X-band position of the fundamental (F), the high-field (H), and the low-field (L) modes versus the nominal thickness $t_{\mathrm{Si}}$. Inset shows a typical $\mathrm{X}$-band FMR spectrum for a $[30 \AA \mathrm{Fe} / 13 \AA \mathrm{Si}]_{50}$ multilayer. Lines are guides to the eye only.

acoustic-like mode, sensitively depends on coupling strength at X-band frequency, i.e., in the non-saturated magnetic state. Using the dispersion relations for the acoustic-like fundamental mode [8] that has all the magnetic layers precessing in phase, we determined the saturation field $H_{\mathrm{S}}=4 H_{\mathrm{E}}$ ( $H_{\mathrm{E}}$ is the exchange field) whose behavior on $t_{\mathrm{Si}}$ is nearly the same as that determined from $H_{\mathrm{S}}$ of hysteresis loops (Fig. 2). We find such an approach useful for evaluating with FMR the coupling field strength in strongly AF coupled multilayers.

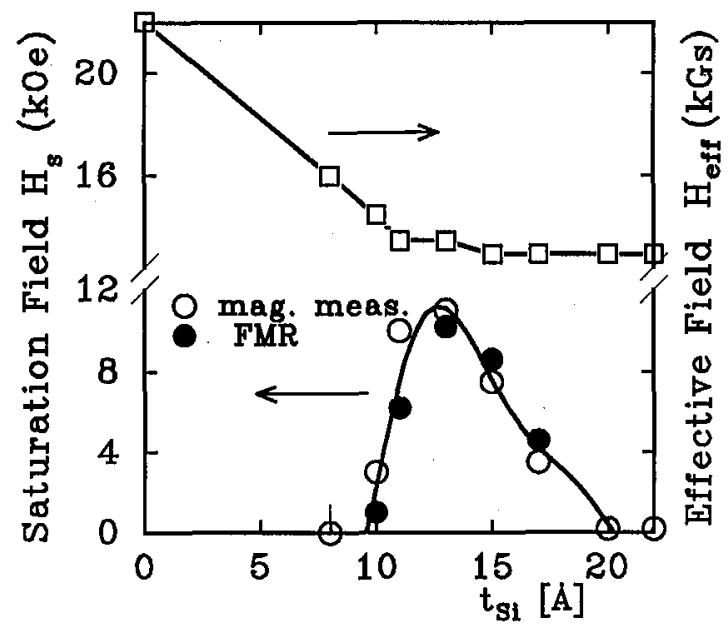

Fig. 2. The saturation field (circles) and the effective field (squares) versus $t_{\mathrm{Si}}$ in $\left[30 \AA \mathrm{Fe} / t_{\mathrm{Si}} \mathrm{Si}\right]_{50}$ multilayers. The values of $H_{\mathrm{S}}$ were determined from hysteresis loops and from FMR X-band data. The values of $H_{\text {eff }}$ were determined from $Q$-band FMR data. 
The spectra taken at $Q$-band, where the magnetization configuration is in a nearly saturated state, consist of only one mode characteristic of an acoustic-like mode degenerated with that of the single layer ferromagnetic resonance [8]. According to the dispersion relations in the saturated state, the position of the fundamental mode at $Q$-band is determined by the effective field $H_{\text {eff }}=4 \pi M_{\mathrm{S}}$ $2 K_{\mathrm{U}} / M_{\mathrm{S}}$, where $K_{\mathrm{U}}$ is the perpendicular anisotropy. In Fig. 2 it is seen that $H_{\text {eff }}$ takes the bulk value of $22 \mathrm{kGs}$ for $t_{\mathrm{Si}}=0 \AA$, decreases with increasing $t_{\mathrm{Si}}$, and above $t_{\mathrm{Si}}=13 \AA$ saturates at a constant value of about $13 \mathrm{kGs}$. Assuming that $K_{\mathrm{U}}$ is negligible, such a behavior suggests magnetization of ferromagnetic layers diminishing with increasing $t_{\mathrm{Si}}$ due to alloying effects.

In summary, we have investigated the strongly $\mathrm{AF}$ coupled $\mathrm{Fe} / \mathrm{Si}$ layered structures in which the enhanced diffusion between $\mathrm{Fe}$ and $\mathrm{Si}$ creates defected non-magnetic crystalline silicide interfaces and partially alloyed ferromagnetic $\mathrm{Fe}$ sublayers. Dispersion relations for AF coupled bilayer structures, frequently used for analyzing FMR in multilayers, are shown to be inapplicable to the $\mathrm{Fe} / \mathrm{Si}$ multilayers with a strong AF coupling and a substantial biquadratic component. We have found, however, that the AF coupling strength can be evaluated from the position of the fundamental mode at X-band frequency.

\section{Acknowledgments}

The authors would like to thank Prof. K. Röll from Kassel University, Germany for use of their deposition apparatus.

\section{References}

[1] H. Fredrikze, A. van der Graaf, M. Valkier, F.J.A. den Broeder, Physica B 234-236, 498 (1997).

[2] J.J. de Vries, J. Kohlhepp, F.J.A. den Broeder, R. Coehroorn, R. Jungblut, A. Reinders, W.J.M. de Jonge, Phys. Rev. Lett. 78, 3032 (1997).

[3] J. Kohlhepp, F.J.A. den Broeder, M. Valkier, A. van der Graaf, J. Magn. Magn. Mater. 165, 431 (1997).

[4] J. Kohlhepp, M. Valkier, A. van der Graaf, F.J.A. den Broeder, Phys. Rev. B 55, R696 (1997).

[5] E. Fullerton, S.D. Bader, Phys. Rev. B 53, 5112 (1996).

[6] A. Chaiken, R.P. Michel, M.A. Wall, Phys. Rev. B 53, 5518 (1996).

[7] K. Inomata, S.N. Okuno, Y. Saito, K. Yusu, J. Magn. Magn. Mater. 156, 219 (1996).

[8] P.E. Wigen, in: Magnetic Multilayers, Eds. L.H. Bennett, R.E. Watson, World Scientific, Singapore 1994, p. 183. 